\title{
Status of the KM3NeT Mediterranean Sea neutrino telescope
}

\author{
Paschal Coyle ${ }^{1, *}$ \\ ${ }^{1}$ Aix-Marseille Univ., CNRS/IN2P3, CPPM, Marseille, France
}

\begin{abstract}
The status and physics performance of the KM3NeT neutrino telescope in the Mediterranean Sea neutrino are presented.
\end{abstract}

\section{Introduction}

KM3NeT is a large European research infrastructure that will consist of a network of deepsea neutrino detectors in the Mediterranean Sea [1]. The project was selected on the 2016 ESFRI roadmap and will facilitate scientific breakthroughs in the following areas:

- In neutrino physics: KM3NeT will enable ground breaking measurements of the physics of neutrino oscillations, with as flagship measurement the determination of the neutrino mass hierarchy.

- In neutrino astronomy: KM3NeT will be the largest telescope of its kind. Its superior angular resolution will allow for true neutrino astronomy. It will enable the identification of the sources of cosmic neutrinos, the measurement of their energy spectra and the study of the flavour composition of the fluxes.

- In marine biology, Earth and sea research: KM3NeT will offer the opportunity for long-term, high-bandwidth, continuous data taking of environmental parameters. Moreover, these data can be correlated with the data from the neutrino telescope, which also tracks luminescent biological activity (see Ref. [2] for more details).

In the next decade the KM3NeT facility will be one of the few places in Europe where neutrino oscillations and cosmic neutrinos/rays can be studied. As such, it will provide unique opportunities to European (astroparticle) physicists, astronomers and marine scientists alike.

As neutrinos only partake in the weak interaction, their interaction cross section with matter is very small compared to that of photons. Detecting cosmic neutrinos therefore requires enormous $\left(\mathrm{km}^{3}\right)$ volumes, which can only be realised economically by using natural bodies of water (or ice). When these rare interactions between a neutrino and a nucleus in the water occur, charged particles are produced. These particles create light via the Cherenkov

${ }^{*}$ Corresponding author: coyle@cppm.in2p3.fr 
mechanism, which can be detected by a 3D array of light sensors. The direction, energy and type of the neutrino can then be reconstructed from the recorded data. The deep waters of the Mediterranean Sea thus serve both as target for the neutrinos and detector of their reaction products. The light sensors are photomultiplier tubes hosted in glass spheres that resist the water pressure and are transparent to the Cherenkov light. These "optical modules" will, for the first time in neutrino astronomy, house thirty-one 3-inch PMTs instead of a single large PMT, thereby optimising the photocathode area of an optical module and minimising cost. Eighteen optical modules are arranged along flexible strings kept vertical by buoyancy. The strings are anchored to the sea floor at depths exceeding 2.4 $\mathrm{km}$. The KM3NeT Collaboration will construct with the same technology two different detector arrays at two suitable sites, each targeted at neutrinos in a specific energy range:

- ORCA ("Oscillation Research with Cosmics in the Abyss"): The first array consists of one KM3NeT building block (115 detection units, DU) optimized for the study of atmospheric neutrinos in the energy range 3-100 GeV. Its dense configuration with a vertical spacing of $9 \mathrm{~m}$ and horizontal spacing of $23 \mathrm{~m}$ yields an instrumented volume of 8 Mton. It is being built $40 \mathrm{~km}$ off the coast of Toulon, France at a depth of 2450 $\mathrm{m}$, close to the site of the operating ANTARES neutrino telescope. With ORCA, $\mathrm{KM} 3 \mathrm{NeT}$ will be able to conduct a broad research program in the field of neutrino physics in the era before large, accelerator-based projects such as DUNE and HyperKamiokande come online.

- ARCA (“Astroparticle Research with Cosmics in the Abyss"): The second array, under construction near Cape Passero, Sicily at a depth of $3450 \mathrm{~m}$ and $100 \mathrm{~km}$ from shore, consists of two KM3NeT building blocks (330 detection units). Here a sparse configuration is adopted to optimise the detection of very high-energy (TeV-PeV) neutrinos. The vertical spacing between the optical modules within the DU is $36 \mathrm{~m}$, and the horizontal distance between the DUs is $90 \mathrm{~m}$. This configuration, with an instrumented volume of $1.2 \mathrm{~km}^{3}$, is ideally suited to detect neutrinos of cosmic origin, such as those recently discovered by the IceCube experiment [1]. With ARCA we hope to pinpoint the astronomical origin of these neutrinos with far greater accuracy and for the first time perform all flavour precision astronomy.

\section{Status of KM3NeT}

The construction of the KM3NeT detectors is being vigorously pursued at numerous production sites throughout Europe. As of February 2019 a total of about 450 DOMs have been integrated, sufficient for about 25 detection units. In parallel, the corresponding sea floor networks comprising the main electro-optic cables (MEOC), the junction boxes, and the interlink cables are being installed at the detector sites. In May 2016, the first two ARCA DUs were connected at the KM3NeT-Italy site and in September 2017 the first ORCA detection unit was connected at KM3NeT-France. Both sites were able to acquire data for many months until technical issues with the seafloor networks at both sites prematurely terminated the data taking.

For KM3NeT/ORCA the problem was an electrical short in the MEOC. In September 2018, the MEOC was replaced with a new cable of improved design and a new DU was connected in February 2019. Routine connection of additional DUs is now foreseen for ORCA and a second junction box will be deployed in the summer of 2019. 
In the case of KM3NeT/ARCA the problem was a leak in the junction box. An intervention on the ARCA junction box in January 2019 restored the powering of one of the two detection units. A new design for the ARCA junction boxes is underway and should be operational in the Spring of 2020. The second MEOC for KM3NeT/ARCA should be available on a similar timescale.

\subsection{First data}

The three deployed DUs (ORCA1 and ARCA2) provided high quality data during their period of operation. Figure 1 (left) shows the measured coincidence rate within a time window of $15 \mathrm{~ns}$, of hits/photons on the optical modules as a function of PMT hit multiplicity. For low hit multiplicities the rate is dominated by photons originating from $\mathrm{K}^{\text {to }}$ decays of the salt in the seawater. For multiplicities above six the rate is dominated by photons originating from downgoing muons. As ARCA is deeper than ORCA, its average muon rate is about a factor three lower. Figure 1 (right) shows the rate of $\geq 8$-fold coincidences as a function of depth for both the ORCA and ARCA optical modules. The fitted line is the expected depth dependence from the Bugaev model [4]; good agreement is observed.
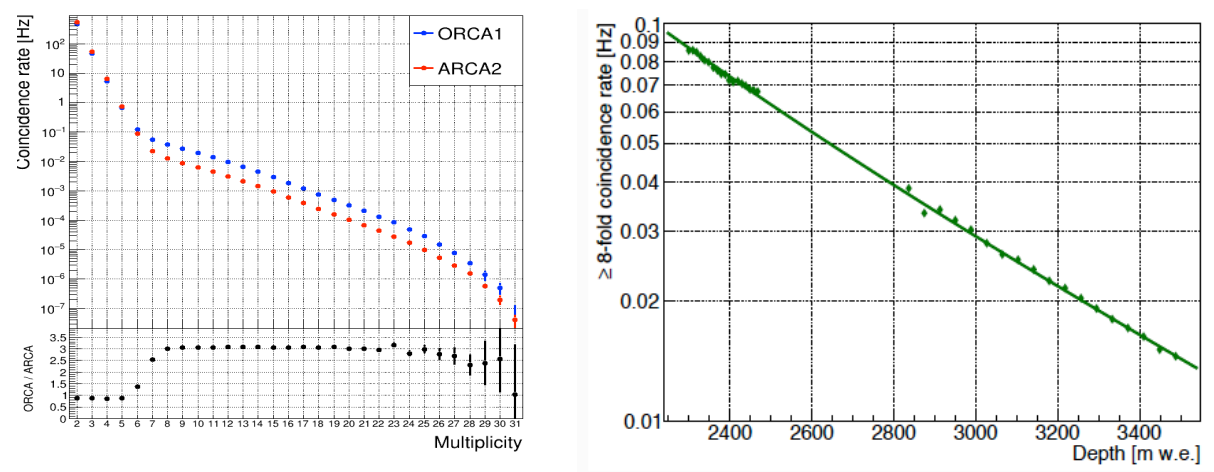

Fig. 1. Left: Coincidence rate as a function of PMT multiplicity for the ORCA1 and ARCA2 detectors averaged over all DOMs of each. Right: Efficiency of $\geq 8$-fold coincidence rates measured with ORCA1 and ARCA2 detectors as a function of depth below sea level fitted the Bugaev parameterisation of the underwater atmospheric muon flux.
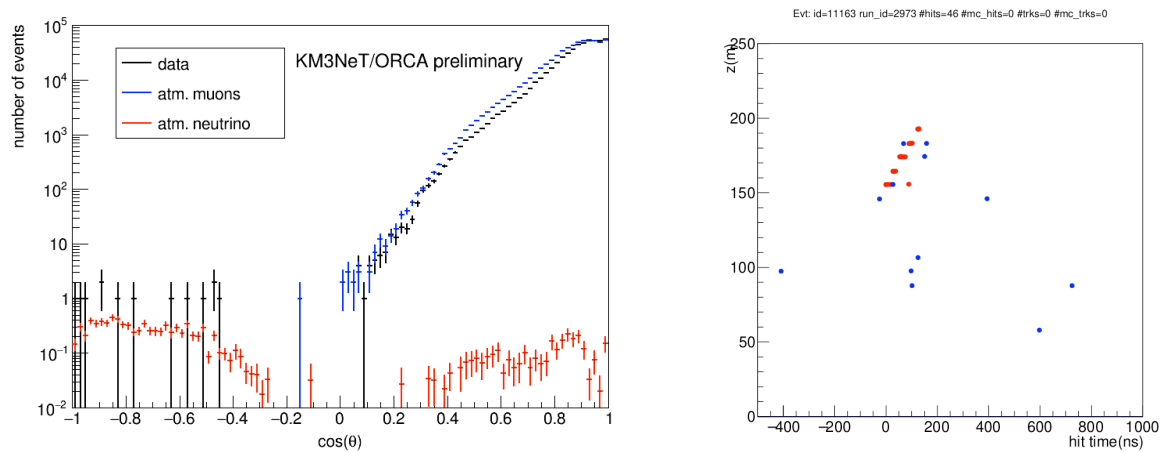

Fig. 2. Left: Zenith angular distribution of track events in the ORCA1 DU; an excess of upgoing events consistent with atmospheric neutrinos is observed. Right: Plot of height versus time for one of the selected neutrino candidates (red hits form the online trigger). 
An analysis has also been performed to isolate atmospheric neutrinos using just the single ORCA DU. Figure 2 (left) shows the reconstructed zenith distribution of the selected track sample. A clear of excess of upgoing tracks is observed, consistent with the predicted flux of atmospheric neutrinos. A height versus time plot of one of the neutrino candidates is shown in Figure 2 (right).

A new idea is being explored to fire a beam of neutrinos from the Protvino accelerator near Moscow to the KM3NET/ORCA detector (see Ref. [5] for details). It turns out this energy/baseline combination is optimal for decoupling the mass hierarchy and CP violation effects. The large instrumented volume of ORCA also allows the intensity of the neutrino beam to be lower than that of conventional long baseline experiments, significantly reducing costs compared to other planned efforts.

\section{Neutrino Astronomy}

The discovery of the existence of a diffuse flux of cosmic neutrinos by the IceCube Collaboration, was one of the main highlights of astroparticle physics in the last decade. It is, of course, of major importance to independently confirm this observation with high significance and very different systematic uncertainties. Figure 3 (left) shows that KM3NeT would provide a greater than 5 sigma observation of the IceCube diffuse flux after only one year of operation; the shower channel providing the highest sensitivity.
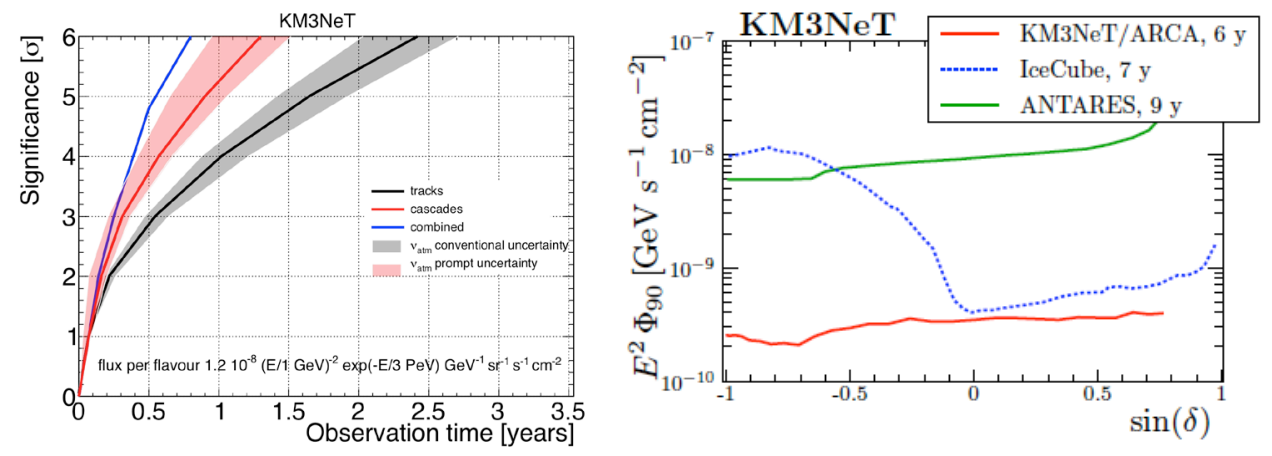

Fig. 3. Left: Significance of KM3NeT/ARCA versus time for the IceCube diffuse neutrino flux. Right: The $90 \%$ C. L. flux sensitivity to a point source of muon neutrinos for ANTARES, IceCube and KM3NeT/ARCA.

Once the diffuse cosmic neutrino flux is confirmed, the major question is the astrophysical origin of these neutrinos. The size of the error ellipse on the arrival direction of a neutrino in KM3NeT/ARCA is typically a factor 20-100 smaller than that of IceCube. For electronneutrinos, for example, the superior optical properties of seawater as a detection medium yield an accuracy of 1.5 degrees at $\mathrm{PeV}$ energies- a factor 10 more accurate than that currently obtained by IceCube. Combining the breakthrough capability of identification all three neutrino types with superior pointing accuracy, will give KM3NeT/ARCA the opportunity to perform genuine neutrino astronomy.

As KM3NeT is located in the Northern Hemisphere it has a complementary field of view to IceCube. Figure 3 (left) shows that the point source sensitivity of KM3NeT/ARCA is an order of magnitude improvement over that of ANTARES in the Southern hemisphere and superior to that of IceCube,. KM3NeT/ARCA has an optimal view of our Galaxy using the high angular resolution upgoing muon track topology. Potential sources of neutrinos in our 
Galaxy include Galactic Pevatrons, micro-quasars, the Fermi Bubbles, neutrinos produced from cosmic ray interactions with molecular clouds. The sensitivity of KM3NeT to the latter source is illustrated in Figure 4. KM3NeT improves by about an order of magnitude over ANTARES and covers a lower energy range than that accessible to IceCube.

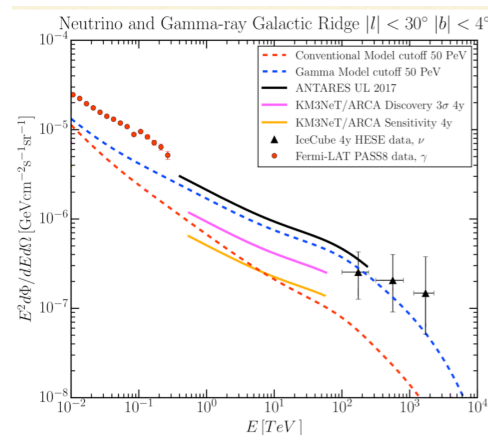

Fig. 4. The neutrino fluxes according to the KRA-gamma model with a $50 \mathrm{PeV}$ energy cutoff (blue dotted line) and the conventional model with the $50 \mathrm{PeV}$ (red dashed line) are shown for all neutrino flavours compared with existing ANTARES and IceCube limits and the expected limits from KM3NeT/ARCA. These expectations and results concern the inner Galactic plane region $\left(111<40^{\circ}\right.$ and $\left.|\mathrm{b}|<3^{\circ}\right)[6]$.

\section{Fundamental neutrino properties}

The oscillation of one neutrino flavour to another is a fascinating quantum mechanical phenomenon at the forefront of current particle physics research. The pattern of the mixing of different mass and flavour states is significantly different from the analogous mixing of quarks. Oscillations tell us about differences between neutrino masses, but not about their actual values. Furthermore, the neutrino mass hierarchy (mass ordering) is a fundamental, but still unknown, parameter of particle physics. Knowledge of its value is essential for the investigation of CP-violation in the neutrino sector. In 2012, when the first measurement of the neutrino mixing angle $\theta_{13}$ was observed to be unexpectedly large, it became clear that the neutrino mass hierarchy could be determined using the neutrinos produced in the Earth's atmosphere by interactions of cosmic rays. It has been shown that this measurement can be performed with the densely instrumented KM3NeT/ORCA detector and could be achieved earlier than with other planned experiments.

To measure the mass hierarchy, the ORCA array will measure about 50,000 neutrinos per year in the energy range between 1 and $100 \mathrm{GeV}$. The study of these neutrinos not only probes the mass hierarchy (Fig. 5, left) with a significance better than 3 sigma in three years, but will also allow for measurements of the $\theta_{23}$ mixing angle, and the mass difference squared between the neutrinos, with a precision exceeding other experiments (Fig. 5, right).

Furthermore, by measuring the number of shower topology events KM3NeT/ORCA can rapidly measure the appearance of the tau neutrino thus providing a test of the unitarity of the neutrino mixing matrix with a precision beyond that of current experiments (Figure 6, left). The latter will establish constraints on neutrinos disappearing into unobserved states ('sterile neutrinos'). The existence of sterile neutrinos can also manifest through their effect on the neutrino oscillations. Figure 6 (right) shows the KM3NeT/ORCA sensitivity to two sterile couplings. 

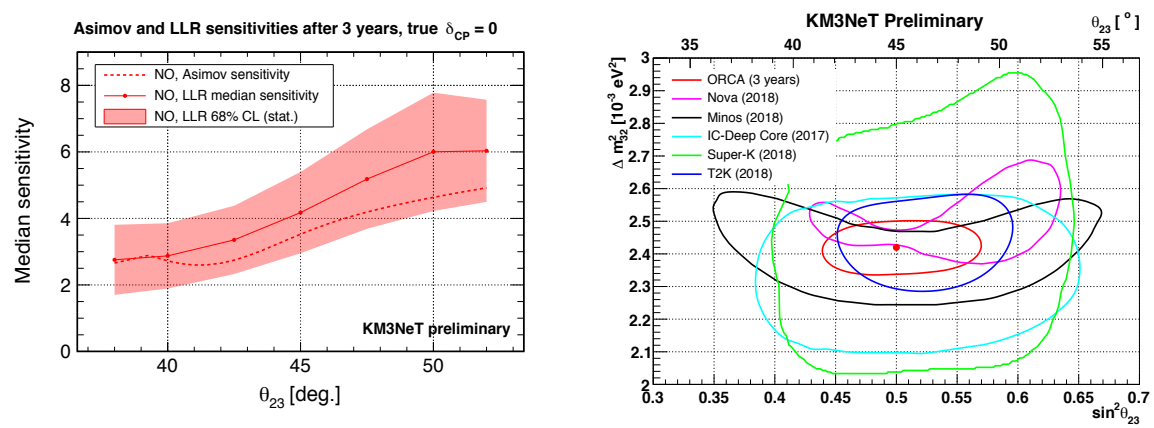

Fig. 5. (left) Median sensitivity in sigma of KM3NeT/ORCA to the neutrino mass ordering as a function of $\theta 23$. (right) Constraints on the $\theta 23$ mixing angle and the mass squared mass difference as a function of $\theta 23$. (Both plots for 3 years of operation).

Thanks to the facetted optical module design, all three building blocks (i.e. ARCA+ORCA) will have sensitivity to the neutrinos produced in Galactic supernovae. These neutrinos, with energies around $10 \mathrm{MeV}$, cause coincident signals on the sensors of a single optical module, leading to an $80 \%$ chance to detect a Galactic supernova if one occurs, yielding invaluable information on our understanding of supernovae explosions and neutrino masses alike.
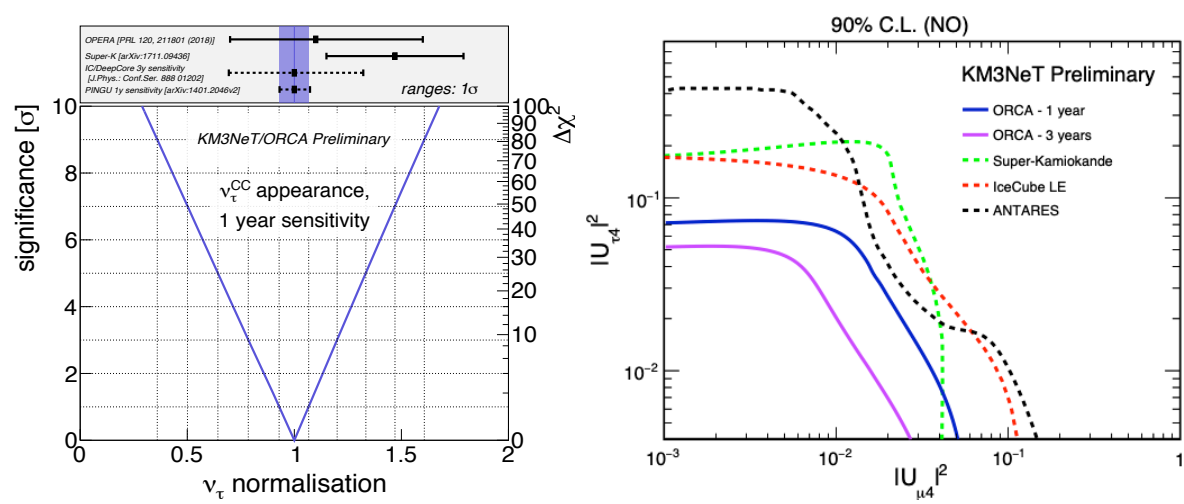

Fig. 6. Left: Precision on the neutrino tau normalisation after 1 year of data taking. Right: Constraints on the couplings to a sterile neutrino from ANTARES, IceCube and KM3NeT/ORCA.

\section{References}

1. S. Adrian-Martinez et al. (KM3NeT Collaboration.), J. Phys. G: Nucl Part. Phys. 43 (2016) 084001.

2. D. LeFevre, these proceedings.

3. M. G. Aartsen et al. (IceCube Collaboration), Science 361 (2018) 147-151.

4. E. V. Bugaev et al., Phys. Rev. D 58 (1998) 054001.

5. J. Brunner, Alexandre Zaitsev, these proceedings.

6. A. Marinelli, adapted from Phys. Rev. D 96 (2017) 062001. 